\title{
Co-infección por los virus del dengue y chikungunya. Revisión narrativa
}

\author{
Sandra Lucía Vargas', Diana Carolina Céspedes², Juan David Vergel, \\ Erika Paola Ruiz y María Camila Luna
}

\footnotetext{
'Facultad de Medicina, Universidad El Bosque (UEB), Bogotá, Colombia. ${ }^{2}$ Estudiante Facultad de Medicina Universidad El Bosque (UEB) Bogotá, Colombia.

Los autores no registran conflicto de interés alguno en este artículo.

Recibido: 6 de noviembre de

Aceptado: 20 de septiembre de 2018

Correspondencia a: Sandra Lucía Vargas Cruz vargassandra@unbosque.edu.co
}

(a)

\begin{abstract}
Coinfection of dengue and chikungunya viruses. Narrative review
Background: Dengue and chikungunya viruses are transmitted by the female Aedes aegypti and Aedes albopictus, which are widely distributed in tropical and subtropical areas, facilitating coinfection. Aim: To determine the magnitude, geographical distribution and clinical picture of dengue and chikungunya coinfection. Material and Methods: Narrative review. A search in the PubMed and Lilacs databases was made, using the MeSH terms "Chikungunya", "CHIKV", "DENV", "Dengue" and "coinfection. The articles of the last 20 years were included. Results: A total of 45 articles were included. The largest coinfection report was in Asia followed by Africa. In the Americas, the information is limited because of the recent circulation of chikungunya. The magnitude of coinfection varies between $0 \%$ and $31.9 \%$. No differences were found in the distribution of coinfection by sex and age. The clinical picture of monoinfection and coinfection was similar. Some case reports show severe cases with central nervous system involvement, hemorrhagic manifestations and Still's disease. Conclusion: The clinical manifestations of coinfection by dengue and chikungunya viruses are similar to those due to monoinfection, which difficult the diagnosis and measurement of its magnitude.
\end{abstract}

Keywords: Dengue; chikungunya; coinfection; Aedes aegypti; Aedes albopictus.

Palabras clave: Dengue; Chikungunya; co-infección; Aedes aegypti; Aedes albopictus.

\section{Introducción}

$\mathrm{L}$ a fiebre del dengue y el chikungunya son conocidas como enfermedades infecciosas distribuidas en zonas tropicales y subtropicales de todo el mundo, caracterizadas principalmente por fiebre, artralgias y mialgias ${ }^{1}$.

El virus del dengue (DENV) pertenece a la familia Flaviviridae, género Flavivirus, formando parte de los arbovirus, es decir, virus transmitidos por artrópodos; posee cuatro serotipos, DENV-1, DENV-2, DENV-3 y DENV-4².

El virus del chikungunya (CHIKV) pertenece a la familia Togaviridae y género Alphavirus, posee un solo serotipo, con tres genotipos diferentes que circulan en el mundo y llevan su nombre según donde fueron detectados por primera vez: el África Occidental (WA), el Este/ Centro/Sur de África (ECSA) y por último, el Asiático ${ }^{1,3}$.

Estos dos virus son transmitidos por la picadura de la hembra de los mosquitos Aedes (Stegomyia) aegypti (Linnaeus, 1762) y Aedes (Stegomyia) albopictus (Skuse, 1894) ${ }^{4-6}$. Ambos mosquitos han colonizado exitosamente muchos sitios, por lo que se consideran especies invasoras y se han expandido ampliamente en el orbe. Aedes aegypti es originario de África mientras que A. albopictus lo es del Oriente e India ${ }^{7,8}$.

El dengue es la enfermedad viral aguda, de más rápida propagación en el mundo, aumentando su incidencia 30 veces en los últimos 50 años $^{2}$. Aproximadamente ocurren 390 millones de infecciones por DENV anualmente, de las cuales 96 millones presentan manifestaciones clínicas, transmitiéndose activamente en 141 países $^{9,10}$. Esto la hace una enfermedad vectorial de especial importancia, con gran impacto en salud pública dadas sus implicaciones en la pérdida de días laborales y su rápida propagación sin respetar límites territoriales ${ }^{2}$.

En las Américas, el dengue mantiene una situación epidemiológica muy compleja, dada la circulación de los cuatro serotipos y las condiciones propicias para su transmisión ${ }^{11}$. En el año 2010 se notificaron más de 1,6 millones de casos de dengue, de los cuales 50.235 correspondían a formas graves, con una mortalidad de 1.185 personas $^{11}$.

La fiebre del chikungunya es una enfermedad endémica en África, Sudeste de Asia e India ${ }^{12}$. Habiéndose aislado el virus por primera vez en Tanzania en 1952, esta enfermedad circuló en África y Asia hasta fines de la década de los 70; luego no fue reportada sino hasta resurgir paulatinamente desde inicios del siglo 21, catalogándosele entonces como una afección re-emergente, ya que des- 
pués de estar en un silencio epidemiológico de casi tres décadas, logró una expansión cada vez mayor a lo largo del mundo ocasionando grandes epidemias y aumentando su gravedad en algunas regiones ${ }^{12,13}$. Se calcula que las tasas de infección en las comunidades afectadas por las epidemias recientes de CHIKV oscilan aproximadamente entre 38 y $68 \%$, para las islas del suroeste del Océano Índico, el Pacífico y el sudeste de Asia ${ }^{14}$.

Según la Organización Mundial de la Salud (OMS), en las Américas, el CHIKV fue detectado por primera vez en diciembre de 2013 en islas del Caribe. Desde junio de 2014, se registró el chikungunya en República Dominicana y desde entonces se han notificado más de 600.000 casos sospechosos, configurándose una amplia expansión a países en riesgo como Costa Rica, El Salvador, Panamá, Venezuela y Colombia, reportando una incidencia de 826 casos por 100.000 habitantes en riesgo para el año $2014^{15}$.

Las enfermedades febriles de etiología viral, incluyendo el dengue y el chikungunya, son la principal causa de enfermedad en los países del trópico. El dengue y el chikungunya pueden co-existir y presentar síntomas similares, lo que dificulta el diagnóstico por parte del personal de salud, ya que, si no se realizan pruebas de laboratorio, difícilmente se puede llegar a diferenciar la presencia de uno o de los dos virus. Además, la falta de medios diagnósticos en los países en vía de desarrollo aumenta la dificultad para estimar la verdadera incidencia de estas enfermedades ${ }^{16}$.

Ambos virus son transmitidos por mosquitos Aedes y están relacionados temporal y espacialmente. La rápida expansión de $A$. aegypti y $A$. albopictus junto con la cocirculación de estos virus, podría conducir a un aumento de la co-infección en humanos, con el riesgo de aumentar la gravedad de la enfermedad. Esta situación justifica la realización de investigaciones sobre la co-infección DENV-CHIKV en temas como su magnitud, distribución mundial, cuadro clínico y gravedad. Además, en América Latina y el Caribe son escasos los estudios de co-infección por DENV y CHIKV, dada su reciente aparición en estos territorios. El objetivo de esta revisión fue determinar la magnitud, distribución geográfica y cuadro clínico de la co-infección por dengue y Chikungunya.

\section{Metodología}

\section{Tipo de estudio}

Revisión narrativa.

\section{Estrategia de búsqueda}

Se realizó la búsqueda de artículos, publicados dentro de los años 1995-2016 en las bases de datos PubMed y LILACS, en las cuales se utilizaron los términos MeSH "Chikungunya", "CHIKV", "DENV", "Dengue" y
"Coinfection". Se empleó el uso del boleano "and", descartando el uso de "or" ya que el interés fue examinar publicaciones sobre la co-infección de DENV y CHIKV. Se utilizaron las siguientes ecuaciones en la base de datos Pubmed: "Dengue" AND "Chikungunya" AND "Coinfection"; "DENV" AND "CHIKV" AND "Coinfection"; "Dengue" AND "Coinfection" AND "Chikungunya”. Además, como complemento, se revisó manualmente las referencias bibliográficas de cada artículo recuperado para incluir otros estudios. Los criterios de inclusión fueron artículos provenientes de todos los países, publicados durante los 20 años anteriores, en los idiomas español e inglés. Se eligieron estudios realizados en humanos y en especies de mosquitos $A$. aegypti o $A$. albopictus. Se excluyeron los artículos que no estudiaron la co-infección por los dos virus o que tuvieran resultados no concluyentes, y realizados en otras especies de mosquitos diferentes a Aedes.

\section{Manejo de la información}

Un grupo de cuatro evaluadores independientes se distribuyeron la lectura de títulos y abstracts. Tras la lectura independiente de cada evaluador se establecía si se incluía o no el artículo para lectura de texto completo; para esto se elaboró una tabla de selección de abstracts. Se elaboraron fichas bibliográficas por cada artículo. La información de cada artículo se registró en un formato previamente establecido por el equipo de investigadores en una base de datos en Microsoft Excel $2010^{\circledR}$ que incluía año y lugar de ocurrencia, población, edad, sexo, pruebas diagnósticas, porcentaje de co-infección, manifestaciones clínicas de la mono-infección y co-infección, hospitalización, mortalidad y filogenética. Posteriormente, se realizó la síntesis de los artículos revisados donde se extrajo finalmente la información incluida en la revisión. Las discrepancias durante la selección de estudios o extracción de datos se resolvieron mediante discusión y consenso.

\section{Definiciones}

La co-circulación está definida como la presencia de dengue y chikungunya en una misma área geográfica, mientras que co-infección es descrita como la detección de ambos virus en un mismo paciente. La co-infección por los virus es confirmada por la presencia de cualquiera de los siguientes criterios:

- ARN de DENV y CHIKV en pacientes con menos de cinco días de inicio de la fiebre.

- Antígeno NS1 específico DENV.

- Anticuerpos específicos de DENV y CHIKV (IgM) en pacientes con cinco o más días de inicio de la fiebre.

- Aumento de cuatro veces en el título de anticuerpos IgG para DENV y CHIKV en muestras pareadas con diferencia de 15 días entre la toma de estas.

- Aislamiento viral. 


\section{Consideraciones éticas}

A partir de la resolución número 8430 de 1993, esta investigación se considera sin riesgo. Además, de acuerdo a la ley 23 de 1983, se respetaron los derechos de autor, realizándose una adecuada citación y referenciación de la información de acuerdo a normas de Vancouver.

\section{Resultados}

Se revisó un total de 42 artículos sobre co-infección por DENV y CHIKV en humanos, de los cuales se incluyen 26 estudios de corte transversal, 13 reporte de casos, uno de casos y controles, uno de cohorte prospectivo y una revisión narrativa. Además, se analizaron cuatro artículos sobre co-infección en vectores, para un total de 45 artículos incluidos.

\section{Magnitud y distribución de la co-infección}

De los artículos revisados, 19 fueron de Asia, 10 en África, 12 en las Américas. Cinco estudios reportan casos importados de Angola, La India, América, Nigeria y Singapur publicados en Portugal, Alemania, España, India y Taiwan, respectivamente ${ }^{17-21}$. Se ha informado co-infección en 19 países de África, Asia sudoriental, Mediterráneo oriental, la región del Pacífico occidental y América. Los países que más han publicado sobre co-infección fueron La India y Gabón. En la Figura 1 se muestra la distribución geográfica de la co-infección reportada en los estudios revisados.

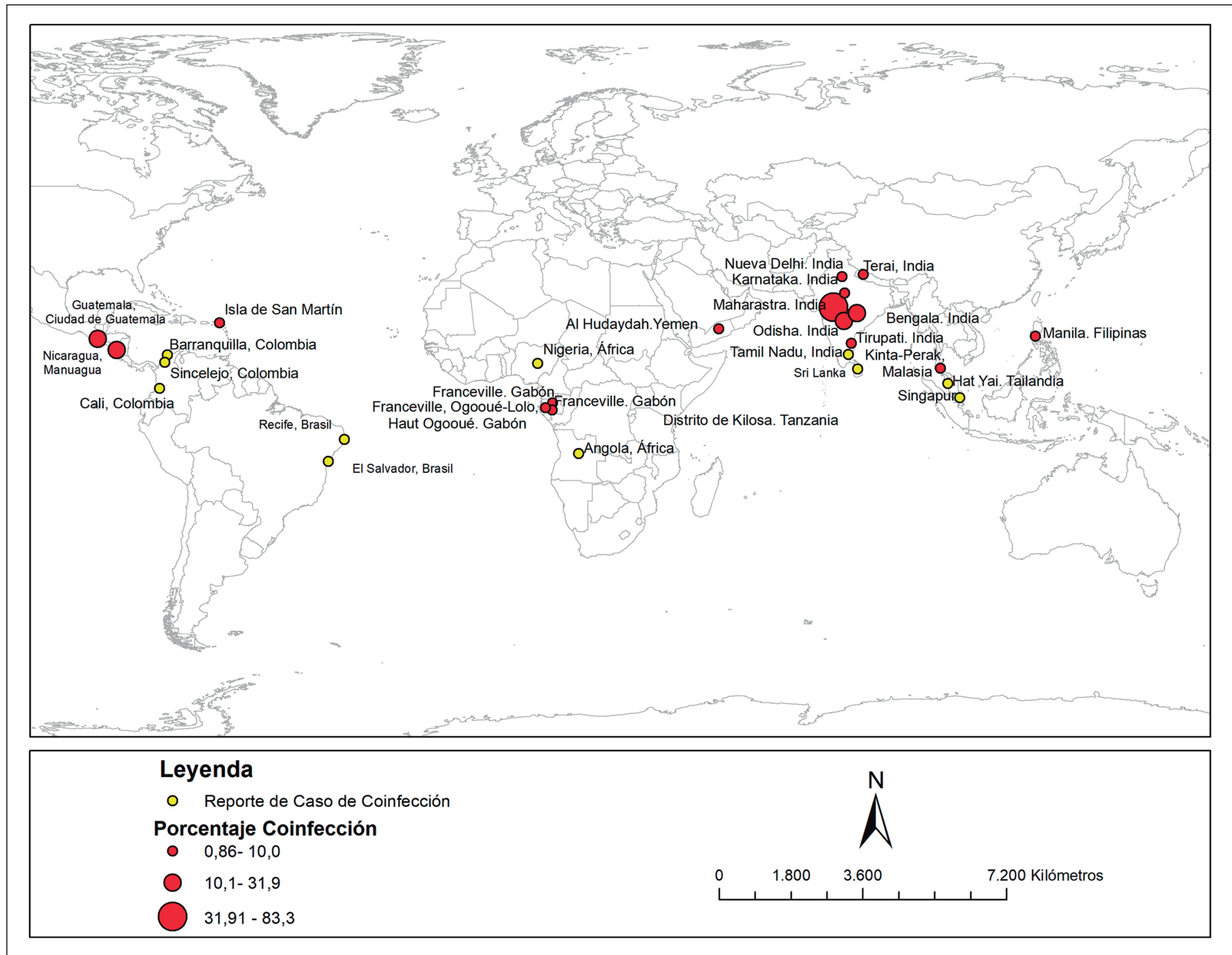

Figura 1. Distribución geográfica de la co-infección por dengue y chikungunya. 
La co-infección por DENV y CHIKV fue reportada por primera vez en 1962 en Tailandia, posteriormente en La India para $1964^{22-24}$ y en Myanmar entre 1970 y $1972^{25}$. Por 34 años, en Asia y África la co-infección no volvió a ser reportada, hasta la aparición de nuevos casos en el 2006 en La India, Madagascar, Sri Lanka, Malasia. En el año 2007 en Gabón, en 2008 en Nigeria, en 2009 en Tailandia y en 2010 en La India ${ }^{26-34}$. En Yemen, en el año 2012, un estudio reportó co-infección de 2,3\% Para el período 2012 y 2013, en Filipinas se identificó la co-infección en 11 casos (10\%) a partir de 112 pacientes con diagnóstico de dengue por reacción de polimerasa en cadena (RPC) ${ }^{36}$. En Nepal, para los mismos años, se analizaron 169 muestras de todos los pacientes febriles al ingreso de varios hospitales y se halló la co-infección en cinco casos $(5,0 \%)^{37}$.

En América, para el 2013 se reportó por primera vez la trasmisión autóctona de CHIKV, en la isla San Martín y en la Guyana Francesa. Desde ese año, más de 44 países en América Central y del Sur han registrado la circulación de $\mathrm{CHIKV}^{38,39}$. Varios estudios han reportado casos de co-infección por DENV y CHIKV en Brasil, Colombia, y publicaciones de la Isla San Martin, de Nicaragua y Guatemala, han informado co-infección de 1,06\%, 14,2\% y $31,9 \%$, respectivamente $38-49$.

De los dos países mencionados que han publicado varios estudios sobre co-infección, en La India, del año 2006 al 2015 reportan co-infección de 2,7 a 13,7\% $\%^{26,34,50-53}$ (Tabla 1). En el año 2013, se registró la co-infección más alta: 13,7\% en 204 muestras de la población de Odisha y $83,3 \%$ en 18 muestras de Maharashtra ${ }^{52}$. En el estado de Karnataka, se estudió la población durante cuatro períodos de tiempo, desde julio de 2010 hasta junio de 2013 , registrando un promedio de co-infección de $8,11 \%$, con un rango de 5,7 a 9,5\%, siendo esta última la magnitud más alta, registrada en el año $2012^{34}$.

En cuanto a Gabón, África, se conoce que ha tenido registro de co-infección desde 2007 hasta el 2010, con magnitudes que alcanzan hasta $5,8 \%{ }^{54}$. Los estudios realizados en varias ciudades de ese país durante el período 2007 a 2010, reportan aumento de los casos de co-infección en los años de brote 2007 y 2010, con casos esporádicos en los demás años ${ }^{1,31,54}$.

En la Tabla 1 se presenta la magnitud de la co-infección reportada en los estudios desde 1970 hasta el año 2016.

Se han evidenciado casos de co-infección en viajeros; en La India, se reportó un joven de 21 años que viajó por un mes a Nigeria, presentando co-infección por DENV, CHIKV y malaria ${ }^{20}$. En Portugal se presentó un caso de una mujer de 50 años que regresó de un viaje a Luanda, Angola, con co-infección por DENV del serotipo $4 \mathrm{y}$ $\mathrm{CHIKV}^{17}$. En Barcelona, España, se realizó un estudio retrospectivo de 42 casos de viajeros provenientes de América, quienes presentaron a su regreso cuadros febriles con artralgias, encontrándose cinco casos de co-infección $(11,9 \%)^{19}$. En Alemania se reportó un caso de co-infección por CHIKV y DENV en una mujer de 25 años, que viajó a La India en el $2008^{22}$. Otro estudio reporta dos casos de co-infección en Taiwán confirmados por serología, tras un viaje a Singapur durante el $2009^{18}$.

Además de la co-infección por DENV y CHIKV, se ha descrito co-infección por virus zika (ZIKV), malaria y salmonela. En Tanzania, África, se encontró co-infección por DENV, CHIKV y malaria en algunos pacientes con síndrome febril, con reporte de $1 \%$ de co-infección por estos virus ${ }^{16}$. En Borno, Nigeria, en 310 pacientes con enfermedad febril se estudió la presencia de Plasmodium falciparum, Salmonella Typhi, CHIKV, fiebre amari1la, DENV y el virus del Nilo occidental. El 8\% de los pacientes fueron negativos para los patógenos estudiados, $25 \%$ cursaban con mono-infección por arbovirus o por P. falciparum y/o Salmonella sp; $20 \%$ presentaba coinfección por DENV-CHIKV y el 44\% restante presentaba co-infección entre dos arbovirus o entre un arbovirus y Plasmodium o Salmonella $\mathrm{sp}^{32}$.

En Brasil, se informó la co-circulación de ZIKV, DENV y CHIKV en el año 2015, y se identificó la presencia de co-infección por DENV y ZIKV en dos pacientes en Tuparetama quienes presentaron exantema, fiebre, conjuntivitis y artralgias, sin reportar co-infección por DENV y CHIKV ${ }^{40}$. En Salvador de Bahía, Brasil, desde el 2015 se identificó la co-circulación de DENV, CHIKV y ZIKV, dificultando el diagnóstico de las enfermedades exantemáticas agudas indiferenciadas; sin embargo, no se reportó co-infección por DENV y CHIKV ${ }^{41}$. En Nicaragua, entre los años 2015 y 2016, se reportó co-infección por DENV, CHIKV y ZIKV en seis pacientes $(1,7 \%)^{48}$.

En cuanto a la distribución de la co-infección por sexo y edad, es poco descrita en los estudios revisados. Dos estudios reportan con mayor frecuencia la co-infección DENV-CHIKV en mujeres, el primero en Gabón con una relación hombre-mujer 0,71:1 y el segundo en Bengala con una relación hombre-mujer $0,58: 1^{1,50}$.

Un estudio realizado en Odisha y Maharastra, La India, reportó diferencias de la co-infección entre hombres y mujeres de acuerdo al grupo de edad, donde fue más frecuente en mujeres entre 31 a 45 años y en hombres entre 16 a 30 años ${ }^{16}$. Otro estudio en La India describió que $91,2 \%$ de los pacientes con co-infección tenían 16 años o más ${ }^{50}$. En Tailandia, un estudio en 50 pacientes de centros de atención pediátrica, en un rango de edad de 1 mes a 15 años, reportó $2 \%$ de co-infección por DENV-CHIKV ${ }^{33}$.

La frecuencia relativa de la co-infección en las áreas urbana y rural es poco descrita en los estudios revisados. Un estudio realizado en Bengala oriental, La India, con un porcentaje de co-infección de 12,4\% describe que $61,7 \%$ de estos casos habitaba en área urbana y semiurbana ${ }^{50}$. 


\begin{tabular}{|c|c|c|c|c|c|c|c|}
\hline Región/país & $\begin{array}{l}\text { Año del } \\
\text { estudio }\end{array}$ & Tipo de estudio & Lugar & n & $\begin{array}{l}\text { Co-infección } \\
\text { DENV-CHIKV (n) }\end{array}$ & $\begin{array}{c}\text { Co-infección } \\
\text { DENV-CHIKV (\%) }\end{array}$ & Referencia \\
\hline \multicolumn{8}{|l|}{ Asia } \\
\hline \multirow[t]{8}{*}{ India } & 2006 & Estudio transversal & Nueva Delhi & 69 & 6 & $8,7 \%$ & 26 \\
\hline & 2010 & Estudio transversal & Bengala Occidental & 550 & 68 & $12,4 \%$ & 50 \\
\hline & 2010- 2012 & Estudio transversal & Estado de Tamil Nadu & 331 & 0 & 0 & 51 \\
\hline & $2010-2013$ & Estudio transversal & Karnataka & 6.554 & 532 & $8,1 \%$ & 34 \\
\hline & 2011 & Estudio Transversal & Nueva Delhi & 87 & 9 & $10,3 \%$ & 69 \\
\hline & 2013 & Estudio transversal & Odisha & 204 & 28 & $13,7 \%$ & 52 \\
\hline & & & Maharashtra & 18 & 15 & $83,3 \%$ & \\
\hline & 2015 & Estudio transversal & Tirupati & 72 & 2 & $2,7 \%$ & 53 \\
\hline Tailandia & 2009 & Estudio de cohorte prospectivo & Hat Yai & 50 & 1 & $2 \%$ & 33 \\
\hline Yemen & 2012 & Estudio transversal & Al Hudaydah & 400 & 13 & $3,25 \%$ & 35 \\
\hline Filipinas & $2012-2013$ & Estudio transversal & Manila & 112 & 11 & $10 \%$ & 36 \\
\hline Nepal & 2013 & Estudio transversal & Región de Terai & 169 & 5 & $5,0 \%$ & 37 \\
\hline \multirow[t]{3}{*}{ Myanmar } & 1970 & Estudio transversal & Rangún & 539 & 36 & $6,7 \%$ & 25 \\
\hline & 1971 & Estudio transversal & Rangún & 129 & 8 & $6,2 \%$ & 25 \\
\hline & 1972 & Estudio transversal & Rangún & 244 & 11 & $4,5 \%$ & 25 \\
\hline Sri Lanka & $2006-2007$ & Estudio transversal & Kandy & 55 & 3 & $5,4 \%$ & 28 \\
\hline \multicolumn{8}{|l|}{ África } \\
\hline \multirow[t]{3}{*}{ Gabón } & 2007 & Reporte de brote & Libreville y otros & 773 & 8 & $1,03 \%$ & 31 \\
\hline & 2007 y 2010 & Estudio transversal & Franceville y otros & 4.287 & 37 & $0,86 \%$ & 1 \\
\hline & 2010 & Reporte de brote & Libreville y Franceville & 343 & 20 & $5,8 \%$ & 54 \\
\hline Nigeria & 2008 & Estudio transversal & Borno, y otros & 310 & 63 & $20,3 \%$ & 32 \\
\hline Tanzania & 2013 & Estudio transversal & Distrito de Kilosa & 364 & 4 & $1 \%$ & 16 \\
\hline Madagascar & 2006 & Estudio transversal & Toamasina & 55 & 10 & $18 \%$ & 27 \\
\hline \multicolumn{8}{|l|}{ América } \\
\hline Caribe & 2013-2014 & Estudio transversal & Isla San Martín & 1.502 & 16 & $1,06 \%$ & 39 \\
\hline $\begin{array}{l}\text { República Dominicana } \\
\text { Venezuela y Colombia }\end{array}$ & 2014-2015 & Estudio retrospectivo & América & 38 & 5 & $13,2 \%$ & 19 \\
\hline Nicaragua & 2015-2016 & Estudio retrospectivo & Managua y otros distritos & 346 & 49 & $14,2 \%$ & 48 \\
\hline Guatemala & 2015 & Estudio restrospectivo & $\begin{array}{c}\text { Ciudad de Guatemala y } \\
\text { otros distritos }\end{array}$ & 144 & 46 & $31,9 \%$ & 49 \\
\hline
\end{tabular}

\section{Manifestaciones clínicas de la co-infección DENV-CHIKV}

Siete estudios no exponen con claridad el cuadro clínico de los pacientes con co-infección en comparación con los pacientes con mono-infección por DENV o CHIKV 26,34,36-37,39,41,53. Dos artículos en La India y uno en Guatemala presentan con mayor precisión el cuadro clínico de los pacientes co-infectados.

El primer artículo en el año 2009, referido al estado de Bengala Occidental, en pacientes de 1 a 70 años de edad, reportó la fiebre como la manifestación más frecuente de la mono-infección y de la co-infección (100\%); la fiebre bifásica se encontró en todos los casos con co-infección. La diarrea se informó sólo en pacientes con co-infección $(16,2 \%)$. La artralgia intensa e inflamación de las articulaciones eran comunes sólo en los casos con CHIKV, siendo rara en los pacientes con co-infección. El dolor abdominal se asoció principalmente con la infección por DENV. Las artralgias, las erupciones cutáneas, la cefalea, las mialgias y el vómito se reportan, tanto en co-infección como en mono-infección ${ }^{50}$.

El segundo artículo fue realizado en Orisha y Maha- 
rashtra, donde se estudiaron 204 pacientes entre los 0 y 60 años. Los pacientes con co-infección tenían fiebre, cefalea, artralgias, mialgias y trombocitopenia ${ }^{52}$.

El tercer estudio, realizado en Guatemala, expone que las características clínicas de los pacientes con mono-infecciones y co-infecciones fueron similares. Los pacientes con co-infección presentaron porcentajes más altos de rash cutáneo que los que tenían sólo dengue y mayor hospitalización en comparación con los que tenían mono-infección por $\mathrm{CHIKV}^{49}$.

\section{Gravedad y mortalidad de la co-infección DENV-CHIKV}

Un reporte de caso realizado en el año 2008 describe una paciente alemana que viajó a La India. A su regreso presentó fiebre, artralgias, mialgias y cefalea, posteriormente rash cutáneo generalizado, eritema facial, linfadenopatías axilares e inguinales dolorosas, diarrea, vómito y metrorragia prolongada ${ }^{18}$. Otra publicación proveniente de La India, identificó manifestaciones hemorrágicas en tres pacientes co-infectados, uno de los cuales presentó hemorragia en el sistema nervioso central ${ }^{52}$. Adicionalmente, un estudio en Brasil, reportó un caso de una paciente de 27 años, con co-infección y complicaciones neurológicas, las que se resolvieron completamente después de dos meses ${ }^{42}$.

En Cali, Colombia, para el año 2015 se reportaron dos casos de síndrome hiperferritinémico. El primero, asociado únicamente a infección por CHIKV, desarrolló un síndrome antifosfolípidos catastrófico y el segundo, asociado a co-infección DENV-CHIKV, desarrolló la enfermedad de Still en el adulto (AOSD) ${ }^{45}$.

Respecto a la mortalidad, un estudio de casos y controles realizado en La India, en la ciudad de Pune, reportó más mortalidad en los casos de co-infección DENVCHIKV en comparación con los de mono-infección, 12 versus $2 \%$, respectivamente. Dos de los pacientes coinfectados requirieron ventilación mecánica y transfusión de sangre ${ }^{55}$. Otro estudio en Colombia, reportó seis casos fatales en adultos con DENV y CHIKV en el período 2014 y 2015. Cuatro casos se presentaron en personas mayores de 60 años y un caso en una mujer embarazada ${ }^{47}$.

Con relación al cuadro clínico en la población pediátrica, sólo un estudio, realizado en una población de un mes a 15 años de edad, describió la sintomatología de la mono-infección por DENV y CHIKV. Este estudio identificó las artralgias como el síntoma principal de infección por CHIKV, asociadas a rash cutáneo en casi $90 \%$ de los niños, en comparación con los que padecían únicamente DENV, quienes tuvieron mialgias sin artralgias o rash cutáneo en 33,3 a 50\% de los casos. Adicionalmente, se encontró una menor duración de la fiebre en la infección por CHIKV. Sólo un paciente resultó co-infectado y aunque no se describe el cuadro clínico de la co-infección, reportan que la gravedad del cuadro clínico no aumentó ${ }^{50}$.

\section{Co-infección en el vector y su distribución geográfica}

Los principales vectores de CHIKV y DENV, $A$. aegypti y A. albopictus, circulan predominantemente en el continente Asiático y Americano ${ }^{56}$. La distribución de los mosquitos Aedes ha cambiado rápidamente ${ }^{31}$. Por ejemplo, en Colombia la mayor altitud registrada para $A$. aegypti ha sido de $2.302 \mathrm{msnm}^{6}$. Aedes albopictus especie nativa de Asia, proveniente de la India, con los años ha invadido Europa, África y América ${ }^{1,5,38,57}$.

Del total de 42 estudios que reportan co-infección en humanos, sólo cuatro hacen referencia al estudio entomológico ${ }^{1,23,27,31}$ y cuatro se refieren a la co-infección en mosquitos $^{1,57-59}$. De 164 países con presencia de $A$. aegypti y 88 países con $A$. albopictus, 68 reportan la presencia simultánea de las dos especies de vectores ${ }^{38}$.

Aedes albopictus circula predominantemente en zonas rurales y selváticas, mientras que $A$. aegypti circula predominantemente en zonas urbanas, aunque algunos autores afirman que el crecimiento urbano y las movilizaciones poblacionales, como la creación de nuevas vías de comunicación, han permitido la dispersión de este vector hacia áreas rurales, facilitando la entrada del DENV y el CHIKV a estas zonas ${ }^{31,60-61}$. Se ha reportado, en consecuencia, la presencia de los dos vectores en zonas rurales y urbanas, así como la presencia de $A$. aegypti en zonas rurales de Colombia, Brasil, Venezuela y Cuba ${ }^{60-64}$. Un estudio en Colombia detectó los cuatro serotipos de DENV en mosquitos de áreas rurales de dos municipios ${ }^{65}$.

En zonas donde $A$. aegypti no se encuentra presente, como en algunas ciudades de China, Japón o Hawaii, el vector transmisor de CHIKV y DENV es A. albopictus. Incluso se ha visto que la dispersión del $A$. albopictus a territorios colonizados por A. aegypti estuvo acompañada por un brote de DENV-2 ${ }^{31,58}$. Algunos autores afirman que la expansión geográfica de A. albopictus contribuyó a un aumento en la co-infección de estos virus y en la aparición de los brotes ${ }^{1,31}$.

La co-infección DENV-CHIKV en los mosquitos se ha evidenciado en el laboratorio y en condiciones naturales. In vitro, dos estudios demostraron co-infección en $A$. albopictus $^{57-58}$. Mientras que, otro estudio, en el que se infectaron 119 hembras de A. aegypti con los virus del CHIKV y DENV por medio de una membrana artificial, no reportó co-infección ${ }^{59}$.

La co-infección de $A$. albopictus por DENV y CHIKV in vivo se identificó en el brote de Gabón, África, en el año $2010^{1}$. En Gabón, para el mismo año, se halló una mutación en A226V del vector A. albopictus como una posible adaptación, que pudo influir también en que éste haya sido el único vector en el que se halló la coinfección ${ }^{31}$. Con lo anterior surgen dos posibilidades, la primera es que un mismo paciente haya presentado la coinfección como resultado de la picadura de dos mosquitos 


\begin{tabular}{|c|c|c|c|c|}
\hline Región/país & Año & Serotipo DENV & Genotipo CHIKV & Referencia \\
\hline \multicolumn{5}{|l|}{ América } \\
\hline Brasil & 2015 & $1,2,3$ & No reporta & (40) \\
\hline Américas & 2014-2015 & No reporta & Asiático, ECSA & (19) \\
\hline Nicaragua & $2015-2016$ & $1,2,3$ & No reporta & (48) \\
\hline Guatemala & 2015 & $1,2,4$ & No reporta & (49) \\
\hline Colombia & 2014-2015 & 2,3 & No reporta & (47) \\
\hline \multicolumn{5}{|l|}{ Asia } \\
\hline Filipinas & $2012-2013$ & $1,2,3,4$ & Asiático & (36) \\
\hline Yemen & 2012 & 1,2 & No reporta & (35) \\
\hline Tailandia & 2009 & No reporta & Asiático & (33) \\
\hline Nueva Delhi, & 2006 & $1,3,4$ & ECSA & (26) \\
\hline India & 2011 & $1,2,3$ & ECSA & (69) \\
\hline \multicolumn{5}{|l|}{ África } \\
\hline Gabón & 2007 & 2 & ECSA & (31) \\
\hline Gabón & 2007-2010 & 2 & $\begin{array}{c}\text { África Occidental - } \\
\text { ECSA - Asiático }\end{array}$ & (1) \\
\hline
\end{tabular}

diferentes; o la segunda, sería que un mosquito presente la co-infección de estos dos virus y pueda transmitirla al humano tras su picadura ${ }^{31,58}$.

\section{Caracterización filogenética}

La información encontrada acerca del genotipo de DENV y de CHIKV en los artículos revisados es escasa. Doce estudios incluyeron la clasificación filogenética, caracterizados en la Tabla 2.

El CHIKV originario de África posee tres genotipos diferentes: Asia, África occidental y Este/Central/Sur África (ECSA). El CHIKV fue introducido desde África oriental hasta Asia central entre 1940 y 1950, con primeros reportes en la literatura para 1953 en Tanzania ${ }^{66}$. Los brotes en el Océano Índico en el 2004 surgieron de viajeros infectados provenientes de Kenia ${ }^{67}$.

Para el 2006, en Nueva Delhi, desde el sur de La India hasta islas en el Océano Índico, se reportó el genotipo ECSA, mientras que los genotipos aislados en el 2009 en Tailandia podrían corresponder a genotipos asiáticos ${ }^{26,68}$. En La India, para el 2011 se reportó circulación de DENV-1, DENV- 2 y DENV-3, al igual que el genotipo ECSA del CHIKV ${ }^{69}$. En Filipinas, para el período 20122013, a través de RPC para CHIKV, se determinó la presencia del genotipo asiático ${ }^{36}$, similar a lo reportado en viajeros provenientes de las Américas ${ }^{19}$. En Gabón, África, en el 2007 fue encontrado el ECSA, posterior- mente el genotipo asiático y en el 2010 el genotipo de África occidental ${ }^{1,31}$.

En La India, durante el 2013 se analizaron muestras de pacientes co-infectados en un estudio realizado en dos ciudades. Los tipos predominantes de CHIKV y DENV fueron el genotipo ECSA y el serotipo 2, respectivamente. Allí se observaron mutaciones por sustitución (M269V Y D284E) en el gen E1 del CHIKV en 50\% de los pacientes co-infectados. Del mismo modo, se hallaron en la secuencia del gen CprM cinco mutaciones de sustitución, (G70S, L72F, N90S, S93N y I150L) en DENV-1 y dos (A101V y V112A) en DENV-2, detectadas en 44,8\% de los pacientes co-infectados ${ }^{52}$.

\section{Discusión}

En esta revisión se seleccionaron 42 artículos sobre co-infección por DENV y CHIKV en humanos y cuatro artículos sobre co-infección en mosquitos. La magnitud de la co-infección fue variable en los estudios revisados, estuvo entre 1 y $31,9 \%$. Sin incluir el estudio realizado en Maharashtra, el que tuvo una co-infección de 83,3\% en una muestra pequeña de 18 pacientes. Una limitación importante con la interpretación de estos datos es que la prevalencia de la infección monotípica por DENV y CHIKV es muy variable y dinámica en cada país y región ${ }^{38}$. Además, la aparente diferencia en los porcentajes de co-infección pueden ser el resultado de varios factores como la disponibilidad de pruebas diagnósticas en las distintas regiones y países, el desempeño individual de estas pruebas para cada virus, la falta de signos y síntomas para distinguir clínicamente las co-infecciones de las mono-infecciones y las diferencias en los sistemas de vigilancia epidemiológica entre los países, entre otros determinantes ${ }^{38}$.

Además, la comprensión del verdadero alcance de la co-infección por DENV y CHIKV se ve obstaculizada porque el diagnóstico actual se basa, en gran medida, en el cuadro clínico y enfoques distintos de los sistemas de vigilancia epidemiológica en los países. Por ejemplo, el algoritmo de Organización Panamericana de la Salud (OPS) recomienda el examen clínico de especímenes mediante el uso de ensayos consecutivos y la finalización de la detección una vez que se identifica un patógeno; mientras que el Centro para el Control y Prevención de Enfermedades (CDC) a su vez indica que se realicen pruebas simultáneas RPC-TR para DENV, CHIKV y ZIKV ${ }^{49}$.

Los resultados de este estudio ponen de relieve la dificultad para el diagnóstico preciso en regiones donde los pacientes pueden estar infectados por cualquiera de estos patógenos y además la dificultad del diagnóstico, ya que el dengue y el chikungunya son enfermedades difíciles de diferenciar por medio del cuadro clínico dado 
que poseen sintomatología similar; se hace necesaria la realización de estudios de laboratorio para la confirmación del diagnóstico ${ }^{36,51}$.

Por lo anterior, en los países en vía de desarrollo es un reto para el personal médico su identificación, ya que el acceso a métodos diagnósticos es limitado ${ }^{16}$. Se requieren pruebas diagnósticas rápidas, sensibles y de bajo costo para DENV, CHIKV y ZIKV, que contribuyan a la detección de casos, la toma de decisiones en cuanto al manejo y seguimiento de los pacientes y la vigilancia en salud pública. Por lo que en las regiones con co-endemia, el diagnóstico de los casos sospechosos es de mayor prioridad.

En relación a la gravedad de la co-infección, en esta revisión se encontraron reportes de casos con compromiso del sistema nervioso central, enfermedad de Still en el adulto y manifestaciones hemorrágicas. Otros estudios reportan aumento en la mortalidad en los casos de coinfección DENV-CHIKV respecto a los pacientes con mono-infección ${ }^{55}$. Al contrario, otros artículos manifiestan que no hay aumento en la gravedad ${ }^{50}$. Sin embargo, todos estos hallazgos deben interpretarse con precaución dado el limitado número de investigaciones clínicas sobre el tema disponibles al día de hoy. Adicionalmente, en la mayoría de estudios no se diferencia si las infecciones por dengue son primarias o secundarias, lo que no permite estudiar con claridad la gravedad de la co-infección.

Además, es importante reconocer que la mayoría de los estudios se han centrado en la detección cualitativa del DENV y CHIKV en suero o plasma, mientras que se han publicado menos datos en relación a la intensidad de la viremia en pacientes con co-infección. Por lo anterior, algunos autores refieren que no se sabe cómo la intensidad de la viremia (carga viral) puede correlacionarse con manifestaciones clínicas o resultados clínicos en estas infecciones ${ }^{48}$.

Una evidencia importante de resaltar en esta revisión es la distribución generalizada de la co-infección por DENV y CHIKV. Estas enfermedades transmitidas por vectores son uno de los principales problemas de salud pública en el mundo, dada su amplia distribución geográfica e intensa transmisión ${ }^{56}$. Las condiciones que podrían favorecer la co-infección por DENV y CHIKV están relacionadas con la alta infestación del vector, la mutación viral que puede aumentar la capacidad vectorial del mosquito, las actividades humanas que favorecen la exposición al vector y la interacción hospedero-vector ${ }^{50,52,58}$. Adicionalmente, las epidemias simultáneas de dengue y chikungunya, han favorecido la oportunidad del vector de infectarse con los dos virus, propiciando la co-infección a través de una sola picadura ${ }^{52}$.

Otro aspecto de importancia epidemiológica es la determinación de la competencia vectorial en las coinfecciones. Los estudios de Furuya y Vazeille, reconocen la relevancia de considerar las diferentes combinaciones de patógeno-vector, planteando que esto se debe estudiar en una resolución más fina serotipo-genotipo debido a la variación en el potencial de transmisión, identificando sinergias y antagonismos en las interacciones de los virus dentro del vector ${ }^{38,58}$.

Entre las limitaciones de este estudio se debe considerar que la búsqueda se limitó a dos bases de datos y solamente en inglés y español, lo que pudo haber generado la exclusión de artículos sobre co-infección. Además, la estimación de la magnitud de la co-infección es limitada ya que la mayoría de estudios no son poblacionales o las muestras no eran representativas. A su vez, se debe tener en cuenta que los estudios seleccionados se caracterizan por una marcada heterogeneidad metodológica, existiendo diferencias en el diseño: la mayoría son estudios observacionales descriptivos, principalmente reportes de caso y estudios de corte transversal. Este hecho representa que los resultados deben ser evaluados con cautela. Por lo tanto, para abordar de una manera óptima el tema, es necesario más estudios, de mejor calidad, con mejor diseño y con mayor potencia estadística y nivel de evidencia.

A partir de los resultados de esta revisión, se puede concluir que la amplia co-circulación mundial de los virus del dengue y chikungunya y la expansión de los vectores Aedes, representan un desafío para el diagnóstico y la atención en salud por el riesgo de co-infección. Por lo que es necesaria una investigación más exhaustiva acerca de la co-infección de estos virus, que permita establecer con exactitud su magnitud, distribución, cuadro clínico y sus implicaciones en la gravedad de la enfermedad, ya que esta información es de gran utilidad para el desarrollo y la implementación de estrategias de prevención, medidas de contención de brotes y un sistema de vigilancia en salud pública que integre la vigilancia epidemiológica con la virológica y entomológica.

El impacto epidemiológico y económico de la coinfección es en gran parte desconocido y rara vez se considera en las estrategias de prevención y control. Además, la co-infección podría tener importantes consecuencias en la patogenicidad y virulencia de los virus, en las manifestaciones clínicas y en la capacidad vectorial dada las mutaciones descritas. Es importante la prevención de la mono-infección DENV o CHIKV, como su co-infección, mediante las medidas de control vectorial y la consulta oportuna a los servicios de salud.

\section{Resumen}

Introducción: Los virus del dengue y chikungunya son transmitidos por la hembra de los mosquitos Aedes aegypti y Aedes albopictus, ampliamente distribuidos en zonas tropicales y subtropicales, lo que facilita la co-infección. 
Objetivo: Determinar la magnitud, la distribución geográfica y el cuadro clínico de la co-infección por dengue y chikungunya. Material y Métodos: Revisión narrativa. Búsqueda en las bases de datos PubMed y Lilacs, utilizando los términos MeSH "Chikungunya", "CHIKV", "DENV", "Dengue" y "coinfection. Se incluyeron los artículos de los últimos 20 años. Resultados: Se incluyeron 45 artículos. El mayor reporte de co-infección fue en Asia seguido de África. En las Américas la información es limitada por la reciente circulación del chikungunya.
La magnitud de la co-infección varió entre 0 y 31,9\%. No se encontraron diferencias en la distribución de la co-infección por sexo y edad. El cuadro clínico de la mono-infección y la co-infección fue similar. Algunos reportes de caso exponen cuadros graves con afección del sistema nervioso central, manifestaciones hemorrágicas y enfermedad de Still. Conclusión: Las manifestaciones clínicas de la co-infección por dengue y chikungunya son similares a la mono-infección, situación que dificulta el diagnóstico y la medición de su magnitud.

\section{Referencias bibliográficas}

1.- Caron M, Paupy C, Grard G, Becquart P, Mombo I, Nso B, et al. Recent introduction and rapid dissemination of chikungunya virus and dengue virus serotype 2 associated with human and mosquito coinfections in Gabon, central Africa. Clin Infect Dis. 2012; 55 (6): 45-53. doi: 10.1093/cid/cis530.

2.- Organización Mundial de la Salud-Programa especial para Investigación y Capacitación en Enfermedades Tropicales. Dengue: Guías para el diagnóstico, tratamiento, prevención y control. Bolivia; 2009. [Fecha de acceso: 07/02/2018] Disponible en: http://www. who.int/denguecontrol/resources/dengue guidelines_2009/es/

3.- Tsetsarkin K, Cheng R, Sherman M, Weaver S. Chikungunya virus: evolution and genetic determinants of emergence. Curr Opin Virol 2011; 1 (4): 310-7. doi: 10.1016/j. coviro.2011.07.004.

4.- Instituto Nacional de Salud (INS), Ministerio de Salud y Protección social. Lineamientos de vigilancia en salud pública, entomológica y de laboratorio ante la introducción del virus Chikungunya en Colombia. Colombia: Bogotá; 2014. [Fecha de acceso: 07/02/2018] Disponible en: https://www.minsalud.gov.co/sites/rid/Lists/ BibliotecaDigital/RIDE/VS/PP/ET/Anexo $\% 20$ $3 \% 20$ Lineamientos $\% 20 \mathrm{de} \% 20$ Vigilancia $\% 20$ CHIKV\%202014.pdf.

5.- Rúa G, Suárez C, Rojo R. Implicaciones epidemiológicas de Aedes albopictus (Skuse) en Colombia. Rev Fac Nac Salud Pública. 2012; 30 (3): 328-37. http://www.scielo.org.co/pdf/ rfnsp/v30n3/v30n3a09.pdf.

6.- Ruiz F, González A, Vélez A, Gómez G, Zuleta L, Uribe S, et al. Presencia de Aedes (Stegomyia) aegypti (Linnaeus, 1762) y su infección natural con el virus del dengue en alturas no registradas para Colombia. Biomédica 2016; 36 (2): 303-8. DOI: https:// doi.org/10.7705/biomedica.v36i2.3301.

7.- Juliano S A, Lounibos L P. Ecology of invasive mosquitoes: effects on resident species and on human health. Ecol Lett 2005; 8 (5): 558-74. DOI: $10.1111 /$ j.1461-0248.2005.00755.

8.- Rey J R, Lounibos P. Ecología de Aedes aegypti y Aedes albopictus en América y transmisión de enfermedades. Biomédica 2015; 35 (2): 177 85. doi: http://dx.doi.org/10.7705/biomedica. v35i2.2514.

9.- Bhatt S, Gething P, Brady O, Messina J, Farlow $\mathrm{A}$, Moyes $\mathrm{C}$, et al. The global distribution and burden of dengue. Nature 2013; 496 (7446): 504-7. doi: [10.1038/nature12060].

10.- Shepard D, Undurraga E, Halasa Y, Stanaway J. The global economic burden of dengue: a systematic analysis. Lancet Infect Dis 2016; 16 (8): 935-41. doi: 10.1016/S14733099(16)00146-8.

11.- Instituto Nacional de Salud. Protocolo de vigilancia en salud publica dengue- Vigilancia y análisis del riesgo en Salud publica grupo de enfermedades trasmisibles. Colombia: Bogotá; 2014. [Fecha de acceso: 07/02/2018] Disponible en: www.disanejercito.mil.co/?idca tegoria $=44454 \&$ download $=$ Y.

12.- Ministerio de Salud de Colombia, Instituto Nacional de Salud. Informe quincenal epidemiológico nacional. Chikungunya en Colombia año 2014. Colombia: Bogotá; 2015: 20 (5): 78-100. [Fecha de acceso: 07/02/2018] Disponible en: http://www.ins.gov.co/buscadoreventos/IQEN/IQEN\%20vol\%2020\%20 2015\%20num\%205.pdf.

13.- Restrepo B. Infección por el virus del Chikungunya. Ces Medicina. 2014; 28(2): 313-23. http://revistas.ces.edu.co/index.php/ medicina/article/view/3067/2224.

14.- Centro para el Control y Prevención de Enfermedades, Organización Panamericana de la Salud. Preparación y respuesta ante la eventual introducción del virus chikungunya en las Américas. Estados Unidos: Washington; 2011. [Fecha de acceso: 07/02/2018] Disponible en: http://www1.paho.org/hq/ dmdocuments/CHIKV_Spanish.pdf.

15.- Organización Panamericana de la Salud. Número de casos reportados de Chikungunya en países o territorios de las Américas 20132014. Estados Unidos; 2015. [Fecha de acceso: 07/02/2018] Disponible en: http:// www.paho.org/hq/index.php?option=com docman\&task $=$ doc_download\&Itemid $=270 \&$ gi $\mathrm{d}=30199$ \&lang $=\mathrm{es}$

16.- Chipwaza B, Mugasa J, Selemani M, Amuri M, Mosha F, Ngatunga S, et al. Dengue and chikungunya fever among viral diseases in outpatient febrile children in Kilosa district hospital, Tanzania. PLoS Negl Trop Dis 2014; 8 (11): 1-11. https://doi.org/10.1371/journal. pntd.0003335.

17.- Parreira E, Centeno S, Lopes A, Portugal D, Constantino A, Nina J. Dengue virus serotype 4 and chikungunya virus coinfection in a traveller returning from Luanda, Angola, January 2014. Euro Surveill. 2014;13; 19 (10): 20730. https:// www.eurosurveillance.org/images/dynamic/ee/ v19n10/art20730.pdf

18.- Schilling S, Emmerich P, Gunther S, SchmidtChanasit J. Dengue and chikungunya virus co-infection in a German traveller. J Clin Virol. 2009; 45: 163-4. doi: 10.1016/j. jev.2009.04.001.

19.- Bocanegra C, Antón A, Sulleiro E, Pou D, Salvador F, Roure S, et al. Imported cases of chikungunya in Barcelona in relation to the current American outbreak. J Travel Med. 2016; 23(3): 1. doi: 10.1093/jtm/tav033.

20.- Raut C, Rao N, Sinha D, Hanumaiah H, Manjunatha M. Chikungunya, dengue, and malaria co-infection after travel to Nigeria, India. Emerg Infect Dis. 2015; 21(5): 908-9. doi: 10.3201/eid2105.141804

21.- Chang S, Su C, Shu P, Yang C, Liao T, Cheng $\mathrm{C}$, et al. Concurrent isolation of chikungunya virus and dengue virus from a patient with coinfection resulting from a trip to Singapore. J Clin Microbiol. 2010; 48 (12): 4586-9. doi: 10.1128/JCM.01228-10.

22.- Nimmannitya S, Halstead S, Cohen S, Margiotta M. Dengue and chikungunya virus infection in man in Thailand, 1962-1964. I. Observations on hospitalized patients with hemorrhagic fever. Am J Trop Med Hyg 1969; 18 (6): 954-71. PMID: 5355242.

23.- Carey D, Myers R, DeRanitz C, Jadhav M, Reuben R. The 1964 chikungunya epidemic at 
Vellore, South India, including observations on concurrent dengue. Trans R Soc Trop Med Hyg. 1969; 63 (4): 434-45. PMID: 5821266.

24.- Myers R, Carey D. Concurrent isolation from patient of two arboviruses, chikungunya and dengue type 2. Science. 1967; 157 (3794): 1307-8. PMID: 6038994.

25.- Khai Ming C, Thain S, Thaung U, Tin U, Myint KS, Swe T, et al. Clinical and laboratory studies on hemorrhagic fever in Burma, 1970-72. Bull World Health Organ 1974; 51(3): 227-35. PMID: 4282477.

26.- Chahar H, Bharaj P, Dar L, Guleria R, Kabra S, Broor S. Co-infections with chikungunya virus and dengue virus in Delhi, India. Emerg Infect Dis 2009; 15 (7): 1077-80. doi: 10.3201/ eid1507.080638.

27.- Ratsitorahina M, Harisoa J, Ratovonjato J, Biacabe S, Reynes J, Zeller H, et al. Outbreak of dengue and chikungunya fevers, Toamasina, Madagascar, 2006. Emerg Infect Dis. 2008; 14(7): 1135-7. doi: 10.3201/eid1407.071521.

28.- Kularatne S, Gihan M, Weerasinghe S, Gunasena S. Concurrent outbreaks of chikungunya and dengue fever in Kandy, Sri Lanka, 2006-07: a comparative analysis of clinical and laboratory features. Postgrad Med J.2009; 85 (1005): 342-6. Doi: 10.1136/ pgmj.2007.066746.

29.- Hapaurachchi H A C, Bandara K B A T, Hapugoda M D, Williams S, Abeyewickreme W. Laboratory confirmation of dengue and chikungunya co-infection. Ceylon Med J 2008; 53 (3): 104-5. PMID: 18982804.

30.- Nayar S, Noridah O, Paranthaman V, Ranjit K, Norizah I, Chem Y, et al. Co-infection of dengue virus and chikungunya virus in two patients with acute febrile illness. Med J Malaysia 2007; 62 (4): 335-6. PMID: 18551940.

31.- Leroy E, Dieudonné N, Becquart P, Ollomo B, Nze-Nkogue C, Moureau G, et al. Concurrent chikungunya and dengue virus infections during simultaneous outbreaks, Gabon, 2007. Emerg Infect Dis 2009; 15(4): 591-3. doi: 10.3201/eid1504.080664

32.- Baba M, Logue C, Oderinde B, Abdulmaleek $\mathrm{H}$, Williams J, Lewis J, et al. Evidence of arbovirus co-infection in suspected febrile malaria and typhoid patients in Nigeria. J Infect Dev Ctries 2013; 7 (1): 51-9. doi: 10.3855/ jidc.2411.

33.- Laoprasopwattana K, Kaewjungwad L, Jarumanokul R, Geater A. Differential diagnosis of chikungunya, dengue viral infection and other acute febrile illnesses in children. Pediatr Infect Dis J 2012; 31: 459-63. doi: 10.1097/INF.0b013e31824bb06d.

34.- Shaikh N, Raunt C, Manjunatha M. Coinfections with chikungunya and dengue viruses: A serological study in Karnataka State, India. Indian J Med Microbiol 2014; 33 (3):
459-60. doi: 10.4103/0255-0857.158607.

35.- Rezza G, El-Sawaf G, Vescio F, Ameri RA, Pomponi A, Metwally D. Co-circulation of dengue and chikungunya viruses, Al Hudaydah, Yemen, 2012. Emerg Infect Dis. 2014; 20(8): 1351-4. doi: 10.3201/eid2008.131615.

36.- Velasco J, Valderama M, López M, Chua J, Latog R, Roque V, et al. Chikungunya virus infections among dengue-like illness patients at a tertiary care hospital in the Philippines, 2012-2013. Am J Trop Med Hyg 2015; 93 (6): 1318-24. doi: 10.4269/ajtmh.15-0332.

37.- Pandey B, Neupane B, Pandey K, Tun M, Morita K. Detection of chikungunya virus in Nepal. Am J Trop Med Hyg 2015; 93 (4): 697 700. doi: [10.4269/ajtmh.15-0092].

38.- Furuya L, Liang S, Milinovich G, Magalhaes $\mathrm{R}$, Clements A, Hu W, et al. Co-distribution and co-infection of chikungunya and dengue viruses. BMC Infect Dis 2016; 16 (84): 188. doi: [10.1186/s12879-016-1417-2].

39.- Omarjee R, Prat C, Flusin O, Boucau S, Tenebray B, Merle O, et al. Importance of case definition to monitor ongoing outbreak of chikungunya virus on a background of actively circulating virus, St Martin, December 2013 to January 2014. Euro Survell 2014; 19 (13): 20753. https://pdfs.semanticscholar.org/8927/4 e57a8eb67770a60c450bb6dff836df8fa59.pdf? $\mathrm{ga}=2.214855831 .143091503 .1543762115$ 126201126.1537990545.

40.- Pessôa R, Patriota J, Lourdes de Souza M, Felix A, Mamede N, Sanabani S. Investigation into an outbreak of dengue-like illness in Pernambuco, Brazil, revealed a cocirculation of zika, chikungunya, and dengue virus type 1 . Medicine 2016; 95 (12): 3201. doi: 10.1097/ MD.0000000000003201.

41.- Cardoso C, Paploski I, Kikuti M, Rodrigues M, Silva M, Campos G, et al. Outbreak of exanthematous illness associated with zika, chikungunya, and dengue viruses, Salvador, Brazil. Emerg Infect Dis 2015; 21 (12): 2274-6. doi: 10.3201/eid2112.151167.

42.- Brooks J B, Ruiz C A, Fragoso Y D. Acute illness with neurological findings caused by coinfection of dengue and chikungunya viruses in a Brazilian patient. J Infect Public Health 2017; 10 (3): 359-60. doi: 10.1016/j. jiph.2016.08.010.

43.- Villamil-Gómez W E, Rodríguez-Morales A J, Uribe-García A M, González-Arysmendi E, Castellanos J E, Calvo E P, et al. Zika, dengue, and chikungunya co-infection in a pregnant woman from Colombia. Int J Infect Dis 2016; 51: 135-8. doi: 10.1016/j.ijid.2016.07.017.

44.- Villamil-Gómez W E, González-Camargo O, Rodríguez-Ayubi J, Zapata-Serpa D, Rodríguez-Morales A J. Dengue, chikungunya and zika coinfection in a patient from Colombia. J Infect Public Health 2016; 9: 6846. doi: 10.1016/j.jiph.2015.12.002.
45.- Betancur J, Navarro E, Echeverry A, Moncada P, Cañas C, Tobón G. Hyperferritinemic syndrome: Still's disease and catastrophic antiphospholipid syndrome triggered by fulminant chikungunya infection: a case report of two patients. Clin Rheumatol 2015; 34(11): 1989-92. doi: 10.1007/s10067-015-3040-9.

46.- Hoz J, Bayona B, Viloria S, Accini J, Juan-Vergara H, Viasus D. Fatal cases of chikungunya virus infection in Colombia: diagnostic and treatment challenges. J Clin Virol 2015; 69: 27-9. doi: 10.1016/j. jcv.2015.05.021.

47.- Mercado M, Acosta-Reyes J, Parra E, Pardo L, Rico A, Campo A, et al. Clinical and histopathological features of fatal cases with dengue and chikungunya virus co-infection in Colombia, 2014 to 2015. Euro Surveill. 2016; 21 (22). doi: 10.2807/1560-7917. ES.2016.21.22.30244.

48.- Waggoner J J, Gresh L, Mohamed-Hadley A, Ballesteros G, Davila M J, Tellez Y, et al. Single-reaction multiplex reverse transcription PCR for detection of zika, chikungunya, and dengue viruses. Emerg Infect Dis 2016; 22 (7): 1295-7. doi: 10.3201/eid2207.160326.

49.- Edwards T, Signor L D, Williams C, Donis E, Cuevas L E, Adams E R. Co-infections with chikungunya and dengue viruses, Guatemala, 2015. Emerg Infect Dis 2016; 22 (11): 2003-5. doi: 10.3201/eid2211.161017.

50.- Taraphdar D, Sarkar A, Mukhopadhyay B, Chatterjee S. A comparative study of clinical features between monotypic and dual infection cases with chikungunya virus and dengue virus in west Bengal, India. Am J Trop Med Hyg 2012; 86 (4): 720-3. DOI: 10.4269/ ajtmh.2012.11-0704.

51.- Venkatasubramani K, Paramasivan R, Thenmozhi V, Dhananjeyan K, Balaji T, Jerald S. Diagnosis of chikungunya dominated co-infection with dengue during an outbreak in south India (2010 and 2012). Trop Doct 2015; 45 (3): 197-9. doi: $10.1177 / 0049475515585476$.

52.- Saswat T, Kumar A, Kumar S, Mamidi P, Muduli S, Kumar N, et al. High rates of coinfection of dengue and chikungunya virus in Odisha and Maharashtra, India during 2013. Infect Genet Evol. 2015; 35: 134-41. doi: 10.1016/j.meegid.2015.08.006.

53.- Kalawat U, Sharma K, Reddy S. Prevalence of dengue and chickungunya fever and their co-infection. Indian J Pathol Microbiol. 2011; 54 (4): 844-5. http://www.ijpmonline.org/text. asp?2011/54/4/844/91518.

54.- Nkoghe D, Kassa R, Bisvigou U, Caron M, Grard G, Leroy E. No clinical or biological difference between chikungunya and dengue fever during the 2010 Gabonese outbreak. Infect Dis Rep. 2012; 4 (1): 11-3. doi: 10.4081/ idr.2012.e5. 
55.- Gandhi B, Kulkarni K, Godbole M, Dole S, Kapur S, Satpathy P, et al. Dengue and chikungunya co-infection associated with more severe clinical disease than mono-infection. Int J Health Biomed Res. 2015; 3(3): 117-23. http://ijhbr.com/pdf/April\%202015\%20117123.pdf.

56.- Padilla J, Rojas D, Sáenz R. Dengue en Colombia: Epidemiología de la reemergencia a la hiperendemia. Colombia: Bogotá; 2012. [Fecha de acceso: 07/02/2018] Disponible en: https://www.minsalud.gov.co/sites/rid/ Lists/BibliotecaDigital/RIDE/INEC/INV/ Dengue $\% 20$ en $\% 20$ Colombia.pdf.

57.- Potiwat R, Komalamisra N, Thavara U, Tawatsin A, Siriyasatien P. Competitive suppression between chikungunya and dengue virus in Aedes albopictus C6/36 cell line. Southeast Asian J Trop Med Public Health. 2011; 42 (6): 1388-94. PMID: 22299407.

58.- Vazeille M, Mousson L, Martin E, Failloux A. Orally co-infected Aedes albopictus from La Reunion Island, Indian Ocean, can deliver both dengue and chikungunya infectious viral particles in their saliva. PLoS Negl Trop Dis. 2010; 4 (6): 1-5. https://doi.org/10.1371/ journal.pntd.0000706.

59.- Rohani A, Potiwat R, Zamree I, Lee H.
Refractoriness of Aedes aegypti (Linnaeus) to dual infection with dengue and chikungunya virus. Southeast Asian J Trop Med Public Health 2009; 40 (3): 443-8. PMID: 19842428.

60.- Olano V, Matiz M, Lenhart A, Cabezas L, Vargas S, Jaramillo J, et al. Schools as potential risk sites for vector-borne disease transmission: mosquito vectors in rural schools in two municipalities in Colombia. J Am Mosq Control Assoc 2015; 31(3): 212-22. doi: 10.2987/moco-31-03-212-222.1

61.- Lima-Camara T N D, Honório N A, Lourenço-de-Oliveira R. Frequency and spatial distribution of Aedes aegypti and Aedes albopictus (Diptera, Culicidae) in Rio de Janeiro, Brazil. Cad Saude Publica 2006; 22 (10): 2079-84. PMID: 16951879.

62.- Rubio Y, Guzmán H, Espinoza J, Cárdenas L, Bevilacqua M, Medina D. Primer registro de Aedes (Stegomyia) aegypti (L.) en áreas remotas del Estado Bolívar. Bol Malariol Salud Amb 2011; 51(1): 89-91.

63.- Morales A. Aedes aegypti en zona rural del municipio de La Mesa (Cundinamarca) Colombia, S. A. Biomédica 1981; 1 (4): 223-4. file://C:/Users/pepe/Downloads/1806-7002-1SM\%20(2).pdf.

64.- Marquetti M, Bisset J, Leyva M, García A,
Rodríguez M. Comportamiento estacional y temporal de Aedes aegypti y Aedes albopictus en La Habana, Cuba. Rev Cubana Med Trop 2008; 60 (1): 62-7. http://scielo.sld.cu/pdf/mtr/ v60n1/mtr09108.pdf

65.- Castellanos J, Coronel C. Dengue disease diagnosis: a puzzle to be solved. Rev Fac Med 2014; 62 (4): 617-29. http://dx.doi. org/10.15446/revfacmed.v62n4.45593.

66.- Zeller H, Van W, Sudre B. Chikungunya: Its history in Africa and Asia and its spread to new regions in 2013-2014. J Infect Dis 2016; 214 (5): 436-40. DOI: 10.1093/infdis/jiw391

67.- Kumar N, Gopal S. Reemergence of chikungunya virus in Indian Subcontinent. Indian J Virol 2010; 21 (1): 8-17. doi: [10.1007/ s13337-010-0012-1]

68.- Chalaem P, Chusri S, Fernández S, Chotigeat $\mathrm{W}$, Anguita J, Pal U, et al. Characterization of a chikungunya virus strain isolated from banked patients' sera. Virol J 2016; 13 (1): 150. doi: 10.1186/s12985-016-0606-3.

69.- Afreen N, Deeba F, Khan W, Haider S, Kazim S, Ishrat R. Molecular characterization of dengue and chikungunya virus strains circulating in New Delhi, India Microbiol Immunol 2014, 58: 688-96. doi: 10.1111/13480421.12209 . 